\title{
NOTE ON CERTAIN GROUP ALGEBRAS
}

ALFRED B. WILLCOX

In this note we point out two properties (Theorems 1 and 2) of the group algebra of the direct product of a compact group and a locally compact abelian group and indicate briefly how these properties enable one to extend to these group algebras theorems which are known in the compact and abelian cases. Our interest in these properties is two-fold; they establish the applicability of known results in $[4]^{1}$ to the group algebras in question, and they provide an interesting example of a type of $B$-algebra, a sort of Kronecker product algebra, which may very well deserve independent systematic study. The reader is referred to results of Kaplansky [1] and Segal [2] of which this note is essentially a continuation.

Let $C$ be a compact group, $A$ be a locally compact abelian group, and $G=C \times A$ be their direct product group. We are interested in the group algebras $L^{1}(C), L^{1}(A)$, and $L^{1}(G)$. In particular, we ask how the latter is related to the first two. We will show (1) how the $B$-algebra $L^{1}(G)$ is formed out of the $B$-algebras $L^{1}(C)$ and $L^{1}(A)$ and (2) how the structure space of $L^{1}(G)$ is formed from the structure spaces of $L^{1}(C)$ and $L^{1}(A)$.

Definition 1. The complex algebra $R$ is the Kronecker product of the complex algebras $R_{1}$ and $R_{2}$ (written $R=R_{1} \times R_{2}$ ) if there exists a map $\left(x_{1}, x_{2}\right) \rightarrow x_{1} \times x_{2}$ of $\left(R_{1}, R_{2}\right)$ into $R$ such that

$$
\begin{aligned}
\left(x_{1}+y_{1}\right) \times x_{2} & =x_{1} \times x_{2}+y_{1} \times x_{2}, \\
x_{1} \times\left(x_{2}+y_{2}\right) & =x_{1} \times x_{2}+x_{1} \times y_{2}, \\
\alpha x_{1} \times x_{2} & =x_{1} \times \alpha x_{2}, \quad \alpha \text { complex, } \\
R & =\left\{\sum_{i=1}^{n} x_{1}^{(i)} \times x_{2}^{(i)} \mid n \geqq 1\right\},
\end{aligned}
$$

Either (a) $\sum_{i=1}^{n} x_{1}^{(i)} \times x_{2}^{(1)}=0$ implies either all $x_{1}^{(1)}=0$ or the

(4) $x_{2}^{(0)}$ are linearly dependent or (b) the same dependency condition with the subscripts reversed,

(5) $\left(\sum_{i=1}^{n} x_{1}^{(i)} \times x_{2}^{(i)}\right)\left(\sum_{j=1}^{m} y_{1}^{(j)} \times y_{2}^{(j)}\right)=\sum_{i=1}^{n} \sum_{j=1}^{m} x_{1}^{(i)} y_{1}^{(j)} \times x_{2}^{(i)} y_{2}^{(j)}$.

Presented to the Society April 24, 1954; received by the editors August 3, 1955.

1 Numbers in brackets refer to the bibliography at the end of the paper. 
It is well known that the Kronecker product exists and is unique. We use the same definition in case $R_{1}$ and $R_{2}$ are $B$-algebras, but in this case there is an additional question. Can $R$ be made into a $B$ algebra? We follow Schatten [3] in using the following definition.

Defnition 2. Let $R_{1}$ and $R_{2}$ be $B$-algebras with norms $\|\cdot\|_{1}$ and $\|\cdot\|_{2}$ respectively. If in $R=R_{1} \times R_{2}$ a norm $\|\cdot\|$ can be introduced which satisfies the condition $\left\|x_{1} \times x_{2}\right\|=\left\|x_{1}\right\|_{1} \cdot\left\|x_{2}\right\|_{2}$ for all $x_{1} \in R_{1}$ and $x_{2} \in R_{2}$ and satisfies all the other properties of a norm except completeness then we say that $\|\cdot\|$ is a cross-norm on $R$. The completion of $R$ relative to any cross-norm is called a cross-algebra, $R_{1} \otimes R_{2}$, of $R_{1}$ and $R_{2}$.

Cross-algebras are not unique, of course, and when we write $R_{\mathbf{1}} \otimes R_{\mathbf{2}}$ we shall mean a cross-algebra of $R_{1}$ and $R_{2}$.

THEOREM 1. Let $G_{1}$ and $G_{2}$ be locally compact groups. Then $L^{1}\left(G_{1} \times G_{2}\right)$ $=L^{1}\left(G_{1}\right) \otimes L^{1}\left(G_{2}\right)$.

Proof. Let $f$ be in $L^{1}\left(G_{1}\right)$ and $g$ be in $L^{1}\left(G_{2}\right)$. Then by the Fubini theorem the function $f \times g$ defined by $[f \times g](x, y)=f(x) g(y)$ is in $L^{1}(G)$ where $G=G_{1} \times G_{2}$. Let $K$ be the subset of $L^{1}(G)$ consisting of all finite sums of such elements.

(A) $K=L^{1}\left(G_{1}\right) \times L^{1}\left(G_{2}\right)$ : It will be obvious that $K$ is an algebra if we verify (5) of Definition 1 . We use the Fubini theorem as follows:

$$
\begin{aligned}
\left(f_{1} \times g_{1}\right)\left(f_{2} \times g_{2}\right)(x, y) & =\iint_{G} f_{1}(x h) g_{1}(y k) f_{2}\left(h^{-1}\right) g_{2}\left(k^{-1}\right) d h d k \\
& =\int_{G_{1}} f_{1}(x h) f_{2}\left(h^{-1}\right) d h \cdot \int_{G_{2}} g_{1}(y k) g_{2}\left(k^{-1}\right) d k \\
& =\left(f_{1} f_{2}\right)(x) \cdot\left(g_{1} g_{2}\right)(y) \\
& =\left(f_{1} f_{2} \times g_{1} g_{2}\right)(x, y)
\end{aligned}
$$

so, since convolution in $L_{1}(G)$ is distributive, (5) is satisfied and $K$, being closed under multiplication, is an algebra. (1), (2), and (3) of the definition are obviously satisfied. Now suppose that $F(x, y)$ $=\sum_{i=1}^{n} f_{i} \times g_{i}(x, y)=0$ almost everywhere on $G$, and suppose that $f_{p} \neq 0$ for some $p, 1 \leqq p \leqq n$. It is an easy exercise to see that for almost every $y$ we have $F(x, y)=0$ almost everywhere on $G_{1}$. Choose a set $E$ in $G_{1}$ such that $\int_{E} f_{p}(x) d x \neq 0$. Then $\int_{E} F(x, y) d x=\int_{E} \sum_{i=1}^{n} f_{i}(x) g_{i}(y) d x$ $=\sum_{i=1}^{n} g_{i}(y) \cdot \int_{E} f_{i}(x) d x=\sum_{i=1}^{n} \alpha_{i} g_{i}(y)=0$ almost everywhere on $G_{2}$ and $\alpha_{p} \neq 0$. Thus the $g_{i}$ are linearly dependent in $L^{1}\left(G_{2}\right)$ and (4) is satisfied.

(B) The norm is a cross-norm: 


$$
\begin{aligned}
\|f \times g\| & =\iint|f(x) g(y)| d x d y=\int|f(x)| d x \cdot \int|g(y)| d y \\
& =\|f\|_{1} \cdot\|g\|_{2} \text { by Fubini's theorem. }
\end{aligned}
$$

(C) $K$ is dense in $L^{1}(G)$ : By a rectangle in $G$ we mean a set of the form $E_{1} \times E_{2}$ where $E_{i}$ is a measurable set in $G_{i}$. Clearly the characteristic function of a rectangle is in $K$, for $F_{E_{1} \times E_{2}}(x, y)=f_{E_{1}}(x) g_{E_{2}}(y)$. Also, the characteristic function of the union of two disjoint rectangles is in $K$ since $F_{C \cup D}(x, y)=F_{C}(x, y)+F_{D}(x, y)$ if $C \cap D=0$. Since every measurable set in $G$ can be approximated to within a set of arbitrarily small measure by finite unions of disjoint rectangles, every characteristic function of a measurable set in $G$ can be approximated arbitrarily closely by functions in $K$. This completes the proof since characteristic functions of measurable sets are dense in $L^{1}(G)$.

We will exploit only part of the content of Theorem 1 in the following. A complete understanding of the role of this theorem in the study of group algebras must necessarily await a systematic investigation of the concept of cross-algebras of $B$-algebras. Also not for later use here, but nevertheless interesting, is the fact that in case $G_{1}=C$ is compact and $G_{2}=A$ is locally compact abelian then the center $Z^{1}(G)$ of $L^{1}(G)$ is the closure in $L^{1}(G)$ of $Z^{1}(C) \times L^{1}(A)$ and hence is a cross-algebra of these algebras.

We turn now to question (2) above. Let $R$ be a general $B$-algebra. The collection of maximal regular ideals forms a topological space, called the structure space $S(R)$, under the following topology. If $F$ is a subset of $S(R)$ then the kernel $k(F)$ of $F$ is defined to be $\cap M$, $M \in F$; if $I$ is an ideal in $R$ (we consider only two-sided ideals) the hull $h(I)$ of $I$ is defined to be $\{M \in S(R) \mid I \subset M\}$. The closure $F^{\circ}$ of $F$ is then $h(k(F))$. If $R$ has an identity $S(R)$ is compact, otherwise it is customary to consider the space $S^{\prime}(R)$ formed by adding to $S(R)$ a point $M_{\infty}$ which may be interpreted as being the ideal consisting of all of $R$. A neighborhood of $M_{\infty}$ is $M_{\infty}$ together with the complement in $S(R)$ of a hull whose kernel is regular. $S^{\prime}(R)$ is compact, and $R$ is called a GS-algebra [4] in case $S^{\prime}(R)$ is Hausdorff, or, equivalently, if

(a) $S(R)$ is Hausdorff and

(b) every point in $S(R)$ is contained in an open set whose closure has regular kernel.

If $x$ is in $R$ and $M$ is in $S(R)$ we denote by $x(M)$ the image of $x$ under the natural homomorphism of $R$ onto the difference algebra $R / M$.

Now we return to our special case. We state in condensed form some properties of compact groups $C$, locally compact abelian groups $A$, and their direct products $G$. By $\{C, A, G\}$ we shall mean any one of these groups. The reader is referred to [2] for the following as ap- 
plied to $C$ or $A$ and to [1] for the extensions to $G$. Let $U$ be an irreducible representation of $\{C, A, G\}$ by operators on a $B$-space. Then $U$ is finite dimensional (one dimensional in the case of $A$ ). There is a one-to-one correspondence between these irreducible representations and the maximal regular ideals of $\left\{L^{1}(C), L^{1}(A), L^{1}(G)\right\}$. Under this correspondence the image $M \in S\left(L^{1}\right)$ of $U$ is the kernel of the extension $U_{f}=\int U_{x} f(x) d x$ of $U$ to $L^{1}$. If $\{\hat{C}, \hat{A}, \hat{G}\}$ denotes the collection of these irreducible representations then $\{\hat{C}, \hat{A}, \hat{G}\}$ bears a topology inherited from $S\left(L^{1}\right) . \hat{A}$ is Hausdorff since $L^{1}(A)$ is a commutative regular $B$-algebra and $\hat{C}$ is discrete. If, for $f \in L^{1}$, $M \in S\left(L^{1}\right)$ and $U$ the corresponding element of $\{\hat{C}, \hat{A}, \hat{G}\}, f(M)$ is the image of $f$ in $L^{1} / M$ then the mapping $f(M) \rightarrow U_{f}$ is an isomorphism of $L^{1} / M$ onto the algebra of operators (matrices in this case) $U_{f}$. For our purposes no ambiguity will arise out of a failure to distinguish between $f(M)$ and $U_{f}$. From [1] we have the following: Denote a general element of $C$ by $x$, of $A$ by $y$, and of $G$ by $(x, y)$. If $U_{(x, y)}$ is in $\hat{G}$ then $U_{x} \equiv U_{(x, 1)}$ is in $\hat{C}$ and $U_{y} \equiv U_{(1, y)}$ is in $\hat{A}$. The mapping $U_{(x, y)}$ $\rightarrow\left(U_{x}, U_{y}\right)$ is a one-to-one mapping of $\hat{G}$ onto $\hat{C} \times \hat{A}$. Thus $\hat{G}$, and hence $S\left(L^{1}(G)\right.$ ), possesses a second topology given to it by the topology of $\hat{C} \times \hat{A}$. We shall call this topology on $S\left(L^{1}(G)\right)$ the product topology. Clearly it is Hausdorff. Now let $f$ be in $L^{1}(C)$ and $g$ be in $L^{1}(A)$. Suppose $M \in S\left(L^{1}(G)\right)$ corresponds to $\left(M_{C}, M_{A}\right), M_{C}$ $\in S\left(L^{1}(C)\right), M_{A} \in S\left(L^{1}(A)\right)$ via the mappings $S\left(L^{1}(G)\right) \rightarrow \hat{G} \rightarrow \hat{C} \times \hat{A}$ $\rightarrow S\left(L^{1}(C)\right) \times S\left(L^{1}(A)\right)$. Then we have

$$
\begin{aligned}
(f \times g)(M)= & \iint f(x) g(y) U_{(x, y)} d x d y=\iint f(x) g(y) U_{x} U_{y} d x d y \\
& =\int f(x) U_{x} d x \int g(y) U_{y} d y=f\left(M_{C}\right) g\left(M_{A}\right) .
\end{aligned}
$$

THEOREM 2. The $L^{1}$-algebra of the direct product of a compact group and a locally compact abelian group is a GS-algebra.

Proof. We show that $R=L^{1}(G)$ satisfies conditions (a) and (b) above. Let $F$ be a subset of $S(R)$ of the form $F_{C} \times S\left(L^{1}(A)\right)$ where $F_{C}$ is a closed subset of $S\left(L^{1}(C)\right)$. Let $M^{\prime}$ be in the complement of $F$. If $M^{\prime}$ corresponds to $\left(M_{C}^{\prime}, M_{A}^{\prime}\right)$ then $M_{C}^{\prime}$ is in the complement of $F_{C}$. It is easy to see from the definition of the topology on $S\left(L^{1}(C)\right)$ that there exists an $f \in L^{1}(C)$ such that $f\left(M_{C}\right)=0, M_{C} \in F_{C}$, and $f\left(M_{C}^{\prime}\right) \neq 0$. Let $g \in L^{1}(A)$ be such that $g\left(M_{A}^{\prime}\right)=1$, then

$$
\begin{aligned}
& (f \times g)(M)=f\left(M_{C}\right) g\left(M_{A}\right)=0 \text { for } M \in F, \\
& (f \times g)\left(M^{\prime}\right)=f\left(M_{C}^{\prime}\right) \neq 0 .
\end{aligned}
$$


This means that $M^{\prime}$ is not in the closure of $F$ in $S\left(L^{1}(G)\right)$ so $F$ is closed. Sets of the form $S\left(L^{1}(C)\right) \times F_{A}, F_{A}$ closed in $S\left(L^{1}(A)\right)$, are treated similarly. These sets generate the sets in $S\left(L^{1}(G)\right)$ which are closed in the product topology so we can conclude that the product topology is the weaker of the two. The observation that the product topology is Hausdorff completes the proof that $R$ satisfies (a). Both $L^{1}(C)$ and $L^{1}(A)$ satisfy condition (b) $\left(L^{1}(A)\right.$ because it is regular and $L^{1}(C)$ because in any discrete space a point is a neighborhood of itself). The proof that $L^{1}(G)$ also satisfies this condition is then trivial from the fact that if $f\left(M_{C}\right)=1$ and $g\left(M_{A}\right)=1$ then $(f \times g)\left(M_{C}, M_{A}\right)=1$ where 1 denotes either the scalar 1 or the identity of the appropriate algebra.

We conclude with brief mention of some of the applications of Theorems 1 and 2. In [2] Kaplansky shows that in $L^{1}(G)$ any closed primary ideal is maximal. (An ideal is primary if it is contained in precisely one maximal regular ideal.) This enables us to describe the maximal regular ideals of $L^{1}(G)$ directly in terms of those of $L^{1}(C)$ and $L^{1}(A)$. Let $M \leftrightarrow\left(M_{C}, M_{A}\right)$, then if $I_{C}=M_{C} \times L^{1}(A)$ and $I_{A}$ $=L^{1}(C) \times M_{A}$ it can easily be seen that $\left[I_{C}+I_{A}\right]^{\circ}$ is primary in $M$. Thus $M=\left[I_{C}+I_{A}\right]^{c}$.

Theorem 1 enables us to show that the Wiener Tauberian Theorem in its ideal-theoretic form holds for $L^{1}(G)$. We express this by the statement that $L^{1}(G)$ is Tauberian, i.e., every proper closed ideal has a nonvoid hull.

TheOREM 3. If $R=L^{1}(G)$ then the elements of $R$ which vanish outside compact sets in $S(R)$ form a dense subset of $R$.

Proof. The Theorem holds for $L^{1}(C)$ and $L^{1}(A)$ [2, Theorem 2.6 and Corollary 2.9.1]. An easy argument shows that if $R$ is a GSalgebra then a subset of $S(R)$ is compact if and only if its kernel is a regular ideal in $R . L^{1}(C)$ and $L^{1}(A)$ are $G S$-algebras so if $F_{C}$ is compact in $S\left(L^{1}(C)\right)$ and $F_{A}$ is compact in $S\left(L^{1}(A)\right)$ then there exist $f \in L^{1}(C)$ and $g \in L^{1}(A)$ such that $f\left(M_{C}\right)=1$ on $F_{C}$ and $g\left(M_{A}\right)=1$ on $F_{A}$. As we have observed above, $(f \times g)\left(M_{C}, M_{A}\right)=1$ on $F_{C} \times F_{A}$ so $k\left(F_{C} \times F_{A}\right)$ is regular in $R$. Thus $F_{C} \times F_{A}$ is compact. If $f$ vanishes outside $F_{C}$ and $g$ vanishes outside $F_{A}$ then $f \times g$ vanishes outside $F_{C} \times F_{A}$. The extension of the theorem to $L^{1}(G)$ is then obvious from Theorem 1.

Corollary. $L^{1}(G)$ is Tauberian.

Proof. In [4] it is shown that in a strongly semi-simple GSalgebra the closure of $J(\infty)=\{x \in R \mid x(M)=0$ outside some compact 
set\} is the unique minimal closed ideal with vacuous hull. $L^{1}(G)$ is s.s.s. [2] and Theorem 3 says that $[J(\infty)]^{\circ}=R$.

Finally, a next logical question to ask is whether $I=k(h(I))$ for a closed ideal $I$ with nontrivial hull. It is well known that this is not true in general for group algebras, but since $L^{1}(G)$ is a s.s.s. GSalgebra some information pertaining to this question is available. To conserve space we omit proofs in the following. The results are known in the 1.c. abelian and compact cases and are generalized to $G S$ algebras in [4]. Let $h^{(k)}(I)$ denote the $k$ th derived set of the closed set $h(I)$ in $S(R)$. Let $I$ be a closed ideal in $L^{1}(G)$ such that $h^{(k)}(I)$ is void and $h^{(k-1)}(I)$ is compact (i.e., $h^{(k-1)}(I) \cup\{\infty\}$ is discrete). Then $[k(h(I))]^{k}$ $\subset I \subset k(h(I))$, where $[k(h(I))]^{k}$ is the ideal generated by products of $k$ elements of $k(h(I))$. In particular, $I=k(h(I))$ if $h(I)$ is finite. [4, Corollary 2.4.1].

The last sentence can be sharpened somewhat. The above characterization of the maximal regular ideals in $L^{1}(G)$ leads to the extension from $L^{1}(C)$ and $L^{1}(A)$ to $L^{1}(G)$ of a modified form of Ditkin's condition (D). For $M \in S\left(L^{1}(G)\right)$ there exists a bounded directed set $\left\{h_{\alpha}\right\} \subset J(M) \cap J(\infty)$ such that for any $f \in M, f h_{\alpha} \rightarrow f$. This, together with the facts that $L^{1}(G)$ is a s.s.s. $G S$-algebra and that closed primary ideals are maximal, makes the "Generalized Ditkin" Theorem [4, Corollary 2.6.1] applicable: In $L^{1}(G)$ if the boundary of $h(I)$ contains no perfect subsets then $I=k(h(I))$.

\section{BiBLIOGRAPHY}

1. I. Kaplansky, Primary ideals in group algebras, Proc. Nat. Acad. Sci. U.S.A. vol. 35 no. 3 (1949) pp. 133-136.

2. I. E. Segal, The group algebra of a locally compact group, Trans. Amer. Math. Soc. vol. 61 no. 1 (1947) pp. 69-105.

3. R. Schatten, $A$ theory of cross spaces, Annals of Math. Studies, no. 26, Princeton University Press, 1950.

4. A. B. Willcox, Some structure theorems for a class of Banach algebras, Pacific Journal of Mathematics vol. 6 (1956) pp. 177-192.

YALE UNIVERSITY AND Amherst College 